\title{
A Finite Element Collocation Method for Quasilinear Parabolic Equations
}

\author{
By Jim Douglas, Jr., and Todd Dupont
}

\begin{abstract}
Let the parabolic problem $c(x, t, u) u_{t}=a(x, t, u) u_{x x}+b\left(x, t, u, u_{x}\right), 0<x<1$, $0<t \leqq T, u(x, 0)=f(x), u(0, t)=g_{0}(t), u(1, t)=g_{1}(t)$, be solved approximately by the continuous-time collocation process based on having the differential equation satisfied at Gaussian points $\xi_{i, 1}$ and $\xi_{i, 2}$ in subintervals $\left(x_{i-1}, x_{i}\right)$ for a function $U:[0, T] \rightarrow \mathfrak{H}_{3}$, the class of Hermite piecewise-cubic polynomial functions with knots $0=x_{0}<x_{1}<\cdots<x_{n}=1$. It is shown that $u-U=O\left(h^{4}\right)$ uniformly in $x$ and $t$, where $h=\max \left(x_{i}-x_{i-1}\right)$.
\end{abstract}

1. Introduction. Consider the quasilinear parabolic differential equation

$$
c(x, t, u) \frac{\partial u}{\partial t}=a(x, t, u) \frac{\partial^{2} u}{\partial x^{2}}+b\left(x, t, u, \frac{\partial u}{\partial x}\right), \quad 0<x<1,0<t \leqq T,
$$

subject to the initial condition

$$
u(x, 0)=f(x), \quad 0<x<1,
$$

and the boundary conditions

$$
u(0, t)=g_{0}(t), \quad u(1, t)=g_{1}(t), \quad 0<t \leqq T .
$$

Assume the stability condition

$$
0<m \leqq c(x, t, u) \leqq M, \quad m \leqq a(x, t, u) \leqq M<\infty,
$$

for $0 \leqq x \leqq 1,0 \leqq t \leqq T$, and $-\infty<u<\infty$.

We shall be concerned with the numerical solution of (1.1)-(1.3) by a method of collocation for the particular case in which the approximate solution is an Hermite piecewise-cubic polynomial in the space variable $x$ at each time $t$. More precisely, let

$$
0=x_{0}<x_{1}<\cdots<x_{n}=1, \quad h_{i}=x_{i}-x_{i-1},
$$

and

$$
I_{i}=\left[x_{i-1}, x_{i}\right], \quad I=[0,1]
$$

Let

$$
\mathfrak{H}_{3}=\left\{v=v(x) \in C^{1}(I) \mid v \text { is a cubic polynomial on each } I_{i}, j=1, \cdots, n\right\} .
$$

We shall seek a map $U:[0, T] \rightarrow \mathcal{F}_{3}$ such that $U$ is a good approximation of $u$ for $0 \leqq t \leqq T$. Recall that a basis for $\mathfrak{H C}_{3}$ can be constructed by translation and normalized piecewise-dilation of two functions. Let

Received March 13, 1972.

AMS (MOS) subject classifications (1969). Primary 6568.

Copyright $\odot$ 1973, American Mathematical Society 


$$
\begin{aligned}
& V(x)= \begin{cases}1-3 x^{2}+2 x^{3}, & 0 \leqq x \leqq 1 \\
1-3 x^{2}-2 x^{3}, & -1 \leqq x \leqq 0 \\
0, & |x|>1,\end{cases} \\
& S(x)= \begin{cases}x(1-x)^{2}, & 0 \leqq x \leqq 1 \\
x(1+x)^{2}, & -1 \leqq x \leqq 0 \\
0, & |x|>1\end{cases}
\end{aligned}
$$

Set

$$
\begin{aligned}
& V_{i}(x)= \begin{cases}V\left(\left(x-x_{i}\right) / h_{i+1}\right), & x \geqq x_{i}, \\
V\left(\left(x-x_{i}\right) / h_{i}\right), & x \leqq x_{i},\end{cases} \\
& S_{i}(x)= \begin{cases}h_{i+1} S\left(\left(x-x_{i}\right) / h_{i+1}\right), & x \geqq x_{i}, \\
h_{i} S\left(\left(x-x_{i}\right) / h_{i}\right), & x \leqq x_{i},\end{cases}
\end{aligned}
$$

where $h_{0}=h_{n+1}=1$. Then,

$$
\mathfrak{H}_{3}=\operatorname{Span}\left[V_{0}, S_{0}, \cdots, V_{n}, S_{n}\right]
$$

and $\operatorname{dim}\left(\mathcal{H C}_{3}\right)=2 n+2$. Thus, we need $2 n+2$ relations at each time $t$ to specify the approximate solution $U(t)$. Two of these conditions obviously can be obtained from the boundary conditions; i.e., the coefficients of $V_{0}$ and $V_{n}$ are given by $g_{0}(t)$ and $g_{1}(t)$, respectively. The method of collocation requires that the remaining relations be obtained by having the differential equation satisfied at $2 n$ points. Since there are $n$ intervals $I_{i}$, it seems natural to locate two points in each interval. For reasons associated with approximation theory that will be explained later, we shall choose the points in the following fashion:

$$
\xi_{i, k}=\frac{1}{2}\left(x_{i-1}+x_{i}\right)+(-1)^{k} \frac{h_{i}}{2 \sqrt{ } 3}, \quad j=1, \cdots, n, k=1,2 .
$$

Thus, our collocation method is specified by the equations (the writing of the independent variables $x$ and $t$ being partially suppressed)

$$
\begin{gathered}
\left\{c(U) \frac{\partial U}{\partial t}-a(U) \frac{\partial^{2} U}{\partial x^{2}}-b\left(U, \frac{\partial U}{\partial x}\right)\right\}\left(\xi_{i, k}, t\right)=0, \quad j=1, \cdots, n, k=1,2, \\
U(0, t)=g_{0}(t), \quad U(1, t)=g_{1}(t),
\end{gathered}
$$

for $0<t \leqq T$. In addition, it is necessary to specify initial conditions for $U$; the easiest way to do this if $f \in C^{1}(I)$ is to let $U(x, 0)$ be the $\mathcal{H}_{3}$-interpolant of $f$; i.e., $U(x, 0)$ and $\partial U / \partial x(x, 0)$ agree with $f$ and $f^{\prime}$ at the node points $x_{i}$, respectively.

The object of this paper is to analyze the convergence of the solution $U$ of (1.6) to $u$. Obviously, it is a prerequisite to show that $U$ exists and is unique. We shall demonstrate that there is a constant $C$ depending on $u$ and certain of its derivatives such that

$$
\|u-U\|_{L^{\infty}\left(0, T ; L^{\infty}(I)\right)} \leqq C h^{4}, \quad h=\max _{i} h_{i},
$$


if $U(x, 0)$ is chosen as indicated above. This is optimal order convergence, since $U \in \mathfrak{H}_{3}$. We do not know the minimal smoothness necessary to preserve the fourth order convergence; consequently, we shall not complicate the arguments in this paper by seeking minimal smoothness requirements on $u$.

The placement of the collocation points $\xi_{i, k}$ is critical in obtaining the $O\left(h^{4}\right)$ estimate. For other choices of the collocation points, only second order accuracy is obtained. Obviously, the $\xi_{i, k}$ can be perturbed by terms of $O\left(h^{4}\right)$ without causing a loss in order of accuracy, but such modifications of the method generate no new ideas.

Before practical calculations can be made, it is necessary to discretize (1.6) in time. This can be done in the usual ways, e.g., backward differencing, Crank-Nicolson, etc. The proofs of convergence for these cases can be carried out without great difficulty based on the methods of this paper; however, we shall defer presenting these results so that they can be combined with the extension of the single space variable results to several variables.

It should be noted that Eq. (1.1) was not given in divergence form. There does not seem to be any advantage to having a divergence form for collocation methods, in sharp contrast to Galerkin and finite-difference procedures. It is clear that, under the hypothesis (1.4), no loss of generality in (1.6) results from dividing out the coefficient $a(x, t, u)$, since the arithmetic is unaltered. We shall henceforth assume

$$
a(x, t, u) \equiv 1 .
$$

We can also assume $g_{0}(t)=g_{1}(t)=0$ by modifying $b$ and $c$. For convenience, we shall do so.

The Hermite cubic space $\mathfrak{H C}_{3}$ can be employed in a Galerkin procedure just as readily as in a collocation method. It is also the case that $O\left(h^{4}\right)$ accuracy results [5]. Thus, some comparisons should be offered between the two methods. Practically, the collocation method should run noticeably faster on the computer than Galerkin, given exactly the same nodes. First, there are no quadratures to evaluate in the collocation method. Even with the various methods that have been devised to reduce the effort involved in these quadratures [2], they remain a significant part of any Galerkin calculation. Second, there are only four nonzero coefficients in any of the $2 n$ equations generated by (1.6), while there are six in the Galerkin case. Thus, solving the algebraic systems that result from discretization in time is simpler for collocation. Now, our arguments to be presented below require more smoothness on $u$ for the collocation method than is required for the Galerkin method to obtain the optimal order of convergence. Whether this is real or only a failing of this proof is unknown to us. It is not known whether the same $h$ leads to a smaller error for collocation or Galerkin.

2. Some Preliminary Lemmas. We shall indicate the $L^{2}$ inner products on $I$ and $I_{i}$ as follows (only real functions will arise):

$$
(u, v)=\int_{0}^{1} u v d x=\sum_{i=1}^{n}(u, v)_{i}=\sum_{i=1}^{n} \int_{x_{i-1}}^{x_{i}} u v d x .
$$

It is convenient to define 


$$
\begin{aligned}
\langle u, v\rangle_{i} & =\frac{1}{2}\left(u\left(\xi_{i, 1}\right) v\left(\xi_{i, 1}\right)+u\left(\xi_{i, 2}\right) v\left(\xi_{i, 2}\right)\right) h_{i}, \\
|u|_{i}^{2} & =\langle u, u\rangle_{i}
\end{aligned}
$$

and

$$
\langle u, v\rangle=\sum_{i=1}^{n}\langle u, v\rangle_{i}, \quad|u|^{2}=\langle u, u\rangle .
$$

LEMMA 2.1. For all $f$ and $g$ in $\mathfrak{H}_{3}$,

$$
-\left\langle f^{\prime \prime}, g\right\rangle=\left(f^{\prime}, g^{\prime}\right)-\left.f^{\prime} g\right|_{0} ^{1}+\frac{1}{1080} \sum_{i=1}^{n} f_{i}^{\prime \prime \prime} g_{i}^{\prime \prime \prime} h_{i}^{5},
$$

where $f_{i}^{\prime \prime \prime}$ is the (constant) value of the third derivative of $f$ on $I_{i}$.

Proof. It is sufficient to treat just one interval of length $h_{1}$, since the boundary terms collapse on summation over intervals. Note that the points $\xi_{i, 1}$ and $\xi_{i, 2}$ are exactly the Gaussian quadrature points for the choice of two points and a uniform weight function [1], [4]. Hence, if $p$ is a polynomial of degree three,

$$
\langle p, 1\rangle_{1}=\int_{0}^{h_{1}} p d x .
$$

Thus, if

$$
p(x)=\sum_{i=0}^{3} a_{i} x^{i}, \quad q(x)=\sum_{i=0}^{3} b_{i} x^{i},
$$

then

$$
-\left\langle p^{\prime \prime}, q\right\rangle_{1}=-\int_{0}^{h_{1}} p^{\prime \prime} q d x-\left\langle 6 a_{3} x, b_{3} x^{3}\right\rangle_{1}+\int_{0}^{h_{1}} 6 a_{3} b_{3} x^{4} d x .
$$

A trivial calculation shows that $\left\langle x, x^{3}\right\rangle_{1}=7 h_{1}^{5} / 36$; thus

$$
-\left\langle p^{\prime \prime}, q\right\rangle_{1}=-\int_{0}^{h_{1}} p^{\prime \prime} q d x+\frac{h_{1}^{5}}{1080} p^{\prime \prime \prime} q^{\prime \prime \prime},
$$

and the lemma follows.

It is another easy calculation to see that

$$
\max \left\{\frac{\left(p^{\prime \prime \prime}\right)^{2}}{\int_{0}^{h}\left(p^{\prime}\right)^{2} d x} \mid p \text { a nonconstant cubic }\right\}=\frac{720}{h^{5}}
$$

Thus,

$\frac{1}{1080} \sum_{i=1}^{n} f_{i}^{\prime \prime \prime} g_{i}^{\prime \prime \prime} h_{i}^{5} \leqq \frac{2}{3} \sum_{i=1}^{n}\left\|f^{\prime}\right\|_{L^{2}\left(I_{i}\right)}\left\|g^{\prime}\right\|_{L^{2}\left(I_{i}\right)} \leqq \frac{2}{3}\|f\|_{H_{0^{1}}}\|g\|_{H_{0^{1}}}, \quad f, g \in \mathfrak{K}_{3}$, where

$$
\|f\|_{H_{0}{ }^{2}}^{2}=\int_{0}^{1}\left(f^{\prime}\right)^{2} d x .
$$

The space $H_{0}^{1}=H_{0}^{1}(I)$ is defined as usual [3], along with the other Sobolev spaces. 
Let $\mathfrak{H C}_{3}^{0}$ denote the subspace of $\mathfrak{F}_{3}$ consisting of functions vanishing at $x=0$ and $x=1$.

LEMMA 2.2. If $f \in \mathfrak{H}_{3}^{0}$, then

$$
\|f\|_{H_{0^{1}}}^{2} \leqq-\left\langle f^{\prime \prime}, f\right\rangle \leqq \frac{5}{3}\|f\|_{H_{0^{1}}}^{2} .
$$

This lemma will be quite useful later. It should be noted that, while $\left\langle f^{\prime}, f^{\prime}\right\rangle$ is obviously positive semidefinite on $\mathcal{F}_{3}$, it vanishes for

$$
f(x)=\sum_{i=0}^{n} S_{i}(x) \in \mathfrak{F}_{3}^{0} .
$$

Fortunately, the form that will arise is $-\left\langle f^{\prime \prime}, f\right\rangle$. In fact, we can see by an argument analogous to that of Lemma 2.1 that

$$
\left\langle f^{\prime}, g^{\prime}\right\rangle=\left(f^{\prime}, g^{\prime}\right)-\frac{1}{720} \sum_{i=1}^{n} f_{i}^{\prime \prime \prime} g_{i}^{\prime \prime \prime} h_{i}^{5}, \quad f \in \mathcal{H}_{3},
$$

and

$$
\left|f^{\prime}\right|^{2} \leqq\|f\|_{H_{0^{1}}}^{2}, \quad f \in \mathcal{H}_{3} .
$$

The following lemma is useful in interpreting the error bound that will be derived.

LEMMA 2.3. For $f \in H^{1}$,

$$
\|f\|_{H^{1}}^{2}+|f|^{2} \geqq \frac{1}{4}|| f \|_{H^{1}}^{2} .
$$

(The constant can be of the form $(1+c h)^{-1}$.)

Proof. The relation $f(x)=f\left(\xi_{i, 1}\right)+\int_{\xi_{i, 1}}^{x} f^{\prime}(\tau) d \tau$ implies that

$$
\|f\|_{L^{2}\left(I_{i}\right)}^{2} \leqq 4|f|_{i}^{2}+2 h_{j}^{2}\|f\|_{H_{0^{2}\left(I_{i}\right)}^{2}}^{2},
$$

and

$$
\|f\|_{H^{1}}^{2} \leqq 4\left(\|f\|_{H_{0^{1}}}^{2}+|f|^{2}\right) .
$$

It should be noted that $|f|$ and $\|f\|_{L^{2}}$ are not equivalent with constants independent of the $x_{i}$ 's on $\mathfrak{H}_{3}$ for arbitrary node spacing. This is easily seen by taking a very short interval adjacent to a much longer one. However, it is clear from homogeneity (in $h$ ) and a simple calculation that

$$
|f| \leqq(28 / 27)^{1 / 2}\|f\|_{L^{2}}, \quad f \in \mathcal{H}_{3} .
$$

3. Approximation Theory. We shall bound the error in collocation by first bounding the difference between $U$ and the Hermite cubic interpolant of the true solution $u$. In order to estimate the amount by which the interpolant fails to satisfy the collocation equations (1.6), we shall need a representation of the interpolation error. of $u$ :

For sufficiently smooth functions $u$ on $[0,1]$, define the following interpolants

$$
\begin{aligned}
& T_{3}(u)(x)=u(0) V(x)+u^{\prime}(0) S(x)+u(1) V(x-1)+u^{\prime}(1) S(x-1), \\
& T_{4}(u)(x)=T_{3}(u)(x)+u^{(4)}\left(\frac{1}{2}\right) B(x) \\
& T_{5}(u)(x)=T_{4}(u)(x)+u^{(5)}\left(\frac{1}{2}\right) Q(x)
\end{aligned}
$$


where

$$
B(x)=\frac{1}{4 !} x^{2}(1-x)^{2}, \quad Q(x)=\frac{1}{5 !} x^{2}(1-x)^{2}\left(x-\frac{1}{2}\right)
$$

Using Taylor's theorem with integral remainder and the fact that $T_{i}$ reproduces polynomials of degree less than $i+1$, we obtain by standard techniques [1, pp. 6970], [4, pp. 70-71].

LEMma 3.1. Suppose that, for $l=3$, 4 , or $5, u \in H^{l+1}(I)$. Then

$$
\left(u-T_{l}(u)\right)(x)=\int_{0}^{1} K_{l}(x, t) u^{(l+1)}(t) d t,
$$

where, for each fixed $t$,

$$
K_{l}(x, t)=g_{l}^{(l)}(x)-T_{l}\left(g_{t}^{(l)}\right)(x)
$$

and

$$
g_{t}^{(l)}(x)= \begin{cases}\frac{1}{l !}(x-t)^{l}, & 0 \leqq t \leqq x, \\ 0, & x \leqq t \leqq 1 .\end{cases}
$$

For sufficiently smooth functions $u$ defined on $[0, h]$, define

$$
T_{l, h}(u)(x)=T_{l}(w)(x / h), \quad 0 \leqq x \leqq h, l=3,4,5,
$$

where $w(x)=u(h x)$. Also let

$$
{ }_{h}\langle f, g\rangle=\frac{h}{2} \sum_{i=1}^{2} f g\left(\frac{h}{2}\left(1+(-1)^{i} / \sqrt{ } 3\right)\right), \quad{ }_{h}|f|^{2}={ }_{h}\langle f, f\rangle .
$$

Taking $h=1$ and $e=u-T_{3} u$, we obtain the following relations from Lemma 3.1:

$$
\begin{aligned}
{ }_{1}\left|e^{(k)}\right| & \leqq C\left\|u^{(4)}\right\|_{L^{2}(I)}, \quad k=0,1, \\
{ }_{1}\left|e^{\prime \prime}\right| & \leqq C\left\|u^{(5)}\right\|_{L^{2}(I)}, \\
\left.\right|_{1}\left\langle e^{\prime}, 1\right\rangle \mid & \leqq C\left\|u^{(5)}\right\|_{L^{2}(I)}, \\
\left.\right|_{1}\left\langle e^{\prime \prime}, 1\right\rangle \mid & \leqq C\left\|u^{(6)}\right\|_{L^{2}(I)} .
\end{aligned}
$$

In deriving these relations, we used the facts that

$$
{ }_{1}\left\langle B^{\prime}, 1\right\rangle={ }_{1}\left\langle Q^{\prime \prime}, 1\right\rangle=B^{\prime \prime}(1 / 2 \pm 1 / 2 \sqrt{ } 3)=0 .
$$

Using the relations (3.2) and homogeneity, we obtain the following:

Lemma 3.2. Suppose $u \in H^{6}(0, h)$. Let $e=u-T_{3, h} u$. Then, with a constant $C$ independent of $h$,

$$
\begin{aligned}
{ }_{h}\left|e^{(l)}\right| & \leqq C h^{4-l}\|u\|_{H^{\bullet}(0, h)}, \quad l=0,1, \\
{ }_{h}\left|e^{\prime \prime}\right| & \leqq C h^{3}\|u\|_{H^{*}(0, h)} . \\
\left.\right|_{h}\left\langle e^{\prime}, 1\right\rangle \mid & \leqq C h^{9 / 2}\|u\|_{H^{5}(0, h)}, \\
\left.\right|_{h}\left\langle e^{\prime \prime}, 1\right\rangle \mid & \leqq C h^{9 / 2}\|u\|_{H^{\bullet}(0, h)} .
\end{aligned}
$$


4. A Weighted Galerkin Formulation. In this section, we shall show that the solution of (1.6) can be viewed as the solution of the following Galerkin-type scheme:

$$
\left\langle c(U) U_{t}-U_{x x}-b\left(U, U_{x}\right), Z\right\rangle=0, \quad Z \in \mathcal{F}_{3}^{0} .
$$

In the process of showing this, we establish the existence of the solution of the equivalent systems (1.6) and (4.1) locally in time; the global existence in time then follows from the a priori estimates of the next section.

The form (4.1) is useful for analysis but its time-discrete analogues should not be used in computations, since (1.6) gives schemes that are both more efficient and easier to implement.

Let $\left\{Z_{i}\right\}_{i=1}^{2 n}$ be a basis for $\mathfrak{F}_{3}^{0}$, and write $U(x, t)=\sum \beta_{i}(t) Z_{i}(x)$. The relations given by (1.6) can now be written as

$$
\mathscr{F} \beta^{\prime}+\mathcal{G} \beta=R,
$$

where $\beta=\left(\beta_{1}, \cdots, \beta_{2 n}\right)^{T}$ and $\mathcal{F}=\mathscr{F}(\beta)=\left(f_{i i}\right), \mathcal{G}=\left(g_{i j}\right), R=\left(r_{i}\right)$. If we let $\left\{\xi_{l}^{\prime} \mid l=\right.$ $1, \cdots, 2 n\}=\left\{\xi_{i j} \mid i=1, \cdots, n ; j=1,2\right\}$, then we can express $f_{i i}, g_{i i}$, and $r_{i}$ as

$$
\begin{aligned}
& f_{i j}=c\left(\sum_{l} \beta_{l} Z_{l}\left(\xi_{i}^{\prime}\right)\right) Z_{i}\left(\xi_{i}^{\prime}\right), \\
& g_{i i}=-Z_{i}^{\prime \prime}\left(\xi_{i}^{\prime}\right), \\
& r_{i}=b\left(\sum_{l} \beta_{l} Z_{l}\left(\xi_{i}^{\prime}\right), \sum_{l} \beta_{l} Z_{l}^{\prime}\left(\xi_{i}^{\prime}\right)\right) .
\end{aligned}
$$

In a similar fashion, we can express (4.1) as

$$
\mathfrak{e} \beta^{\prime}+a \beta=s,
$$

where

$$
\begin{aligned}
\mathcal{C}=\mathfrak{e}(\beta) & =\left(c_{i i}\right), \quad a=\left(a_{i j}\right), \quad \delta=\left(s_{i}\right), \\
c_{i i} & =\left\langle c\left(\sum_{l} \beta_{l} Z_{l}\right) Z_{i}, Z_{i}\right\rangle, \\
a_{i j} & =-\left\langle Z_{i}^{\prime \prime}, Z_{i}\right\rangle, \\
s_{i} & =\left\langle b\left(\sum_{l} \beta_{l} Z_{l}, \sum_{l} \beta_{l} Z_{l}^{\prime}\right), Z_{i}\right\rangle .
\end{aligned}
$$

If we can show that the matrix $\mathfrak{e}=\mathfrak{e}(\beta)$ is nonsingular for any $\beta$, it will follow that (4.3) has at most one solution and that (4.3) is solvable locally in time. It follows easily that if $\mathfrak{C}(\beta)$ is nonsingular, then $\mathscr{F}(\beta)$ is nonsingular, since any solution $\tau$ of $\mathcal{F} \tau=0$ would also be a solution of $\mathfrak{e} \tau=0$. Thus, if $\mathfrak{e}(\beta)$ is always nonsingular, (4.2) is also locally solvable in time. Since any solution of (4.2) is clearly a solution of (4.3), we see that they are equivalent.

LeMma 4.1. For any $\beta, \mathrm{e}(\beta)$ is nonsingular.

Proof. Suppose that $\tau, \beta \in \mathbf{R}^{2 n}, \tau \neq 0$, are such that $\mathrm{e}(\beta) \tau=0$. If $U(x)=$ $\sum_{i} \beta_{i} Z_{i}(x)$ and $W(x)=\sum_{i} \tau_{i} Z_{i}(x)$, we see that

$$
\langle c(U) W, W\rangle=0 ;
$$


hence, $W\left(\xi_{k}^{\prime}\right)=0$ for $k=1, \cdots, 2 n$ since $c>0$ for any arguments. Since $W$ is a cubic on $\left[x_{0}, x_{1}\right]$ and vanishes at $x_{0}, \xi_{1,1}, \xi_{1,2}$, either $W \equiv 0$ on $\left[x_{0}, x_{1}\right]$ or $W\left(x_{1}\right) W^{\prime}\left(x_{1}\right)$ $>0$. Now, if $W \equiv 0$ in any $\left[x_{i}, x_{i+1}\right]$, then $W \equiv 0$ on $I$, since in each adjacent interval $W$ would have a double root at the endpoint $\left(W \in C^{1}([0,1])\right)$ and two roots at the collocation points. Thus, since $\tau \neq 0$, we see that $W\left(x_{1}\right) W^{\prime}\left(x_{1}\right)>0$. On $\left[x_{1}, x_{2}\right]$, the quadratic $W^{\prime}$ has roots in $\left(x_{1}, \xi_{2,1}\right)$ and $\left(\xi_{2,1}, \xi_{2,2}\right)$. Hence $W\left(x_{2}\right) W^{\prime}\left(x_{2}\right)>0$. Proceeding inductively, we can see that $W\left(x_{n}\right) W^{\prime}\left(x_{n}\right)>0$, which is a contradiction since $W\left(x_{n}\right)=0$. Thus, the lemma is proved.

5. Convergence Analysis. Let $U$ be the solution of (1.6) where we have assumed without loss of generality that $a(x, t, u)=1$ and $g_{0}(t)=g_{1}(t)=0$; i.e.,

$$
\left\langle c(U) \frac{\partial U}{\partial t}-\frac{\partial^{2} U}{\partial x^{2}}-b\left(U, \frac{\partial U}{\partial x}\right), z\right\rangle=0, \quad z \in \mathcal{F}_{3}^{0} .
$$

Now, assume that $u \in C^{3}(I \times[0, T])$ and let $W:[0, T] \rightarrow \mathfrak{K}_{3}$ be determined at each time $t$ as the $\mathfrak{H}_{3}$-interpolant of $u$. We shall not look at $\zeta=u-U$ directly, but instead we shall estimate $\nu=W-U$ and then apply known approximation theoretical results to $\eta=u-W$. Let

$$
R=\partial^{2} \eta / \partial x^{2} .
$$

Then, it is a straightforward calculation to see that

$$
\begin{aligned}
\left\langle c(U)_{\nu_{t}}-\nu_{x x}, z\right\rangle= & \left\langle[c(U)-c(W)] W_{t}-c(W) \eta_{t}-[c(u)-c(W)] u_{t}+R, z\right\rangle \\
& +\left\langle\left[b\left(u, u_{x}\right)-b\left(W, u_{x}\right)\right]+\left[b\left(W, u_{x}\right)-b\left(W, W_{x}\right)\right], z\right\rangle \\
& +\left\langle\left[b\left(W, W_{x}\right)-b\left(U, W_{x}\right)\right]+\left[b\left(U, W_{x}\right)-b\left(U, U_{x}\right)\right], z\right\rangle \\
= & \left\langle-c_{u}^{*} W_{t} \nu-c(W) \eta_{t}-c_{u}^{* *} u_{t} \eta, z\right\rangle \\
& +\left\langle b_{u}^{*} \eta+b_{u}^{* *} \nu+b_{u_{z}}^{*} \nu_{x}, z\right\rangle \\
& +\langle R, z\rangle+\left\langle b\left(W, u_{x}\right)-b\left(W, W_{x}\right), z\right\rangle,
\end{aligned}
$$

where we have assumed $b$ and $c$ to be differentiable (or at least Lipschitz continuous) with respect to $u$ and $u_{x}$. If these derivatives are bounded, then the choice $z=\nu_{t}$ leads to the inequality

$$
\begin{aligned}
m\left|\nu_{t}\right|^{2}-\left\langle\nu_{x x}, \nu_{t}\right\rangle \leqq & K\left[|\nu|^{2}+\left|\nu_{x}\right|^{2}+|\eta|^{2}+\left|\eta_{t}\right|^{2}\right] \\
& +\frac{m}{3}\left|\nu_{t}\right|^{2}+\left\langle R, \nu_{t}\right\rangle+\left\langle b\left(W, u_{x}\right)-b\left(W, W_{x}\right), \nu_{t}\right\rangle .
\end{aligned}
$$

Add the inequality

$$
\frac{1}{2} \frac{d}{d t}|\nu|^{2} \leqq \frac{m}{6}\left|\nu_{t}\right|^{2}+\frac{3}{2 m}|\nu|^{2}
$$

to obtain

$$
\begin{aligned}
\frac{m}{2}\left|\nu_{t}\right|^{2}+\frac{1}{2} \frac{d}{d t}|\nu|^{2}-\left\langle\nu_{x x}, \nu_{t}\right\rangle \leqq & K\left[|\nu|^{2}+\left|\nu_{x}\right|^{2}+|\eta|^{2}+\left|\eta_{t}\right|^{2}\right] \\
& +\left\langle R, \nu_{t}\right\rangle+\left\langle b\left(W, u_{x}\right)-b\left(W, W_{x}\right), \nu_{t}\right\rangle .
\end{aligned}
$$


Lemma 2.1 implies that

$$
\begin{aligned}
-\left\langle\nu_{x x}, \nu_{t}\right\rangle & =\frac{1}{2} \frac{d}{d t}\left[\left(\nu_{x}, \nu_{x}\right)+\frac{1}{1080} \sum_{j=1}^{n} \nu_{x x x_{i}}^{2} h_{i}^{5}\right] \\
& =-\frac{1}{2} \frac{d}{d t}\left\langle\nu_{x x}, \nu\right\rangle .
\end{aligned}
$$

Integrate over the time interval $(0, t)$. Then,

$$
\begin{aligned}
\frac{m}{2} \int_{0}^{t} & \left|\nu_{t}\right|^{2} d \tau-\frac{1}{2}\left\langle\nu_{x x}, \nu\right\rangle(t)+\frac{1}{2}|\nu|^{2}(t) \\
\leqq & K\left[|\nu|^{2}(0)+\int_{0}^{t}\left(|\nu|^{2}+\left|\nu_{x}\right|^{2}\right) d \tau+\int_{0}^{t}\left(|\eta|^{2}+\left|\eta_{t}\right|^{2}\right) d \tau\right] \\
& -\frac{1}{2}\left\langle\nu_{x x}, \nu\right\rangle(0)+\int_{0}^{t}\left\langle R, \nu_{t}\right\rangle d \tau+\int_{0}^{t}\left\langle b\left(W, u_{x}\right)-b\left(W, W_{x}\right), \nu_{t}\right\rangle d \tau .
\end{aligned}
$$

Integrate by parts in time to get

$$
\int_{0}^{t}\left\langle R, \nu_{t}\right\rangle d \tau=\left.\langle R, \nu\rangle\right|_{0} ^{t}-\int_{0}^{t}\left\langle R_{t}, \nu\right\rangle d \tau
$$

Assume that

$$
\begin{gathered}
\sup _{0<t<T}\|u(t)\|_{H^{\bullet}(I)}=\|u\|_{L^{\infty}\left(0, T^{\prime} ; H^{\bullet}(I)\right)}<\infty, \\
\int_{0}^{T}\left\|u_{t}(t)\right\|_{H^{\bullet}(I)}^{2} d t=\left\|u_{t}\right\|_{L^{2}\left(0, T^{\prime} ; H^{\bullet}(I)\right)}^{2}<\infty .
\end{gathered}
$$

Then

$$
\langle R, \nu\rangle=\sum_{i=1}^{n}\langle R, \nu\rangle_{i}=\sum_{i=1}^{n}\left\langle R, \nu-\bar{\nu}_{i}\right\rangle_{i}+\sum_{i=1}^{n}\left\langle R, \bar{\nu}_{i}\right\rangle_{i}, \quad \bar{\nu}_{i}=h_{i}^{-1}\langle\nu, 1\rangle_{i} .
$$

Recall that the collocation points were chosen so that

$$
\begin{aligned}
\left|\left\langle R, \bar{\nu}_{i}\right\rangle_{i}\right| & =\left|\left\langle\eta_{x x}, \bar{\nu}_{i}\right\rangle_{i}\right| \\
& \leqq K\|u\|_{H^{\bullet}\left(I_{i}\right)} h_{i}^{9 / 2} h_{i}^{-1}\left|\langle\nu, 1\rangle_{i}\right| \\
& \leqq K\|u\| \|_{H^{\bullet}\left(I_{i}\right)} h_{j}^{4}|\nu|_{i},
\end{aligned}
$$

by Lemma 3.2. Thus

$$
|\langle R, \nu\rangle| \leqq \sum_{i=1}^{n}|R|_{i}\left|\nu-\bar{\nu}_{i}\right|_{i}+K \sum_{i=1}^{n}\|u\|_{H^{\bullet}\left(I_{i}\right)} h_{i}^{4}|\nu|_{j}
$$

Since it is trivial (using the elementary version of the Poincaré Lemma) that

$$
\left|\nu-\bar{\nu}_{i}\right|_{i} \leqq K h_{i}|| \nu_{x} \|_{L^{2}\left(I_{i}\right)}
$$

and since

$$
|R|_{i} \leqq K\|u\|_{H^{3}\left(I_{i}\right)} h_{i}^{3}
$$

it follows that 


$$
\begin{aligned}
|\langle R, \nu\rangle| & \leqq K \sum_{i=1}^{n}\left[\|u\|_{H^{8}\left(I_{j}\right)}\left\|\nu_{x}\right\|_{L^{2}\left(I_{j}\right)}+\|u\|_{H^{\bullet}\left(I_{j}\right)}|\nu|_{i}\right] h_{j}^{4} \\
& \leqq \frac{1}{16} \sum_{i=1}^{n}\left(\left\|\nu_{x}\right\|_{L^{2}\left(I_{j}\right)}^{2}+|\nu|_{i}^{2}\right)+K \sum_{i=1}^{n}\|u\|_{H^{\bullet}\left(I_{j}\right)}^{2} h_{i}^{8} \\
& \leqq \frac{1}{16}\left(-\left\langle\nu_{x x}, \nu\right\rangle+|\nu|^{2}\right)+K \sum_{i=1}^{n}\|u\|_{H^{\bullet}\left(I_{j}\right)}^{2} h_{i}^{8} .
\end{aligned}
$$

Note that it follows immediately from the above argument that

$$
\left|\int_{0}^{t}\left\langle R_{t}, \nu\right\rangle d \tau\right| \leqq \frac{1}{16} \int_{0}^{t}\left[-\left\langle\nu_{x x}, \nu\right\rangle+|\nu|^{2}\right] d \tau+K \sum_{i=1}^{n} h_{i}^{8} \int_{0}^{t}\left\|u_{t}\right\|_{H^{\bullet}\left(I_{j}\right)}^{2} d \tau
$$

Also,

$$
\begin{aligned}
& \int_{0}^{t}\left\langle b\left(W, u_{x}\right)-b\left(W, W_{x}\right), \nu_{t}\right\rangle d \tau \\
&=\left.\left\langle b\left(W, u_{x}\right)-b\left(W, W_{x}\right), \nu\right\rangle\right|_{0} ^{t}-\int_{0}^{t}\left\langle\frac{\partial}{\partial t}\left\{b\left(W, u_{x}\right)-b\left(W, W_{x}\right)\right\}, \nu\right\rangle d \tau
\end{aligned}
$$

Now,

$$
\left\langle b\left(W, u_{x}\right)-b\left(W, W_{x}\right), \nu\right\rangle=\sum_{i=1}^{n}\left\langle\left. b_{u_{x}}\left(W, W_{x}\right)\right|_{x=\xi_{i, 1}} \eta_{x}+\psi h_{i} \eta_{x}, \nu\right\rangle_{i},
$$

where $|\psi|$ is bounded independently of $h_{i}$ if the second derivatives of $b$ are bounded. Since the $b_{u_{x}}$ multiplier of $\eta_{x}$ has been reduced to a constant on each $I_{i}$, we can employ the leading orthogonality (3.3) of $\eta_{x}$ to constants on each $I_{i}$ as follows:

$$
\begin{aligned}
\left|\left\langle\left. b_{u_{x}}\right|_{x=\xi_{i, 1}} \eta_{x}, \nu\right\rangle_{j}\right| & \leqq\left|\left\langle\left. b_{u_{x}}\right|_{x=\xi_{i, 1}} \eta_{x}, \bar{\nu}_{i}\right\rangle_{j}\right|+\left|\left\langle\left. b_{u_{x}}\right|_{x=\xi_{i, 1}} \eta_{x}, \nu-\bar{\nu}_{i}\right\rangle_{j}\right| \\
& \leqq K\left[h_{i}^{4}\|u\|_{H^{s}\left(I_{i}\right)}|\nu|_{i}+h_{i}^{4}\left\|\left.u\right|_{H^{s}\left(I_{j}\right)}\right\| \nu_{x} \|_{L^{2}\left(I_{j}\right)}\right] .
\end{aligned}
$$

Also, by (3.3),

$$
\left|\left\langle\psi h_{i} \eta_{x}, \nu\right\rangle_{i}\right| \leqq K h_{i}^{4}\|u\|_{H^{*}\left(I_{j}\right)}|\nu|_{i}
$$

and

$$
\left|\left\langle b\left(W, u_{x}\right)-b\left(W, W_{x}\right), \nu\right\rangle\right| \leqq \frac{1}{16}\left[-\left\langle\nu_{x x}, \nu\right\rangle+|\nu|^{2}\right]+K \sum_{i=1}^{n} h_{i}^{8}\|u\|_{H^{s}\left(I_{j}\right)}^{2} .
$$

Next, note that

$$
\begin{aligned}
\frac{\partial}{\partial t} & {\left[b\left(W, u_{x}\right)-b\left(W, W_{x}\right)\right] } \\
& =\left.\left\{\frac{\partial b}{\partial t}\left(W, \alpha_{x}\right)+\frac{\partial W}{\partial t} \frac{\partial b}{\partial u}\left(W, \alpha_{x}\right)+\frac{\partial^{2} u}{\partial x \partial t} \frac{\partial b}{\partial u_{x}}\left(W, \alpha_{x}\right)+\frac{\partial b}{\partial u_{x}}\left(W, W W_{x}\right) \frac{\partial^{2} \alpha}{\partial x \partial t}\right\}\right|_{\alpha=W} ^{\alpha=u} .
\end{aligned}
$$

It then follows from the argument above that, if $b$ has bounded third derivatives,

$$
\begin{aligned}
& \int_{0}^{t}\left\langle\frac{\partial}{\partial t}\left[b\left(W, u_{x}\right)-b\left(W, W_{x}\right)\right], \nu\right\rangle d \tau \\
& \quad \leqq \frac{1}{16} \int_{0}^{t}\left[-\left\langle\nu_{x x}, \nu\right\rangle+|\nu|^{2}\right] d \tau+K \sum_{i=1}^{n} h_{i}^{8} \int_{0}^{t}\left[\|u\|_{H^{s}\left(I_{j}\right)}^{2}+\left\|u_{t}\right\|_{H^{8}\left(I_{j}\right)}^{2}\right] d \tau .
\end{aligned}
$$


It follows from (5.3)-(5.9), (2.5), and the Gronwall lemma that

$$
\begin{aligned}
& \int_{0}^{T}\left|\nu_{t}\right|^{2} d \tau+\max _{0 \leqq t \leq T}\left[\|\nu\|_{H_{0^{2}}}^{2}+|\nu|^{2}\right] \\
& \leqq K\left[\|\nu(0)\|_{H_{0^{1}}}^{2}+|\nu(0)|^{2}+\sum_{j=1}^{n} h_{j}^{8}\left\{\|u\|_{L^{\infty}\left(0, T ; H^{\bullet}\left(I_{i}\right)\right)}^{2}+\left\|u_{t}\right\|_{L^{2}\left(0, T ; H^{\bullet}\left(I_{j}\right)\right)}^{2}\right\}\right] .
\end{aligned}
$$

Lemma 2.3 and (2.6) imply that

$$
\begin{aligned}
& \int_{0}^{T}\left|\nu_{t}\right|^{2} d \tau+\|\nu\|_{L^{\infty}\left(0, T ; H^{1)}\right)}^{2} \\
& \quad \leqq K\left[\|\nu(0)\|_{H^{1}}^{2}+\sum_{i=1}^{n} h_{j}^{8}\left\{\|u\|_{L^{\infty}\left(0, T ; H^{\bullet}\left(I_{j}\right)\right)}^{2}+\left\|u_{t}\right\|_{L^{2}\left(0, T ; H^{\bullet}\left(I_{j}\right)\right)}^{2}\right\}\right] .
\end{aligned}
$$

In particular,

$$
\begin{aligned}
& \|\nu\|_{L^{\infty}\left(0, T ; L^{\infty}\right)}^{2} \\
& \quad \leqq K\left[\|\nu(0)\|_{H^{1}}^{2}+\sum_{i=1}^{n} h_{j}^{8}\left\{\|u\|_{L^{\infty}\left(0, T ; H^{\bullet}\left(I_{j}\right)\right)}^{2}+\left\|u_{t}\right\|_{L^{2}\left(0, T ; H^{\bullet}\left(I_{j}\right)\right)}^{2}\right\}\right] .
\end{aligned}
$$

Also, it is well known [4] that

$$
\|\eta\|_{L^{\infty}\left(0, T ; L^{\infty}\right)}^{2} \leqq K \sum_{i=1}^{n} h_{j}^{8}\|u\|_{L^{\infty}\left(0, T ; H^{8}\left(I_{j}\right)\right)}^{2} .
$$

Since $u-U=\zeta=\eta+\nu$,

$$
\|u-U\|_{L^{\infty}\left(0, T ; L^{\infty}\right)}^{2}
$$

$$
\leqq K\left[\|\nu(0)\|_{H^{2}}^{2}+\sum_{i=1}^{n} h_{i}^{8}\left\{\|u\|_{L^{\infty}\left(0, T_{i} ; H^{\bullet}\left(I_{j}\right)\right)}^{2}+\left\|u_{t}\right\|_{L^{2}\left(0, T ; H^{\bullet}\left(I_{j}\right)\right)}^{2}\right\}\right] .
$$

If we choose, as is quite natural, to define $U(x, 0)$ as the $\mathcal{H}_{3}$-interpolant of $u(x, 0)$, then

$$
\|u-U\|_{L^{\infty}\left(0, T ; L^{\infty}\right)} \leqq K\left[\|u\|_{L^{\infty}\left(0, T ; H^{\bullet}\right)}+\left\|u_{t}\right\|_{L^{2}\left(0, T ; H^{\bullet}\right)}\right] h^{4},
$$

where

$$
h=\max _{j=1, \cdots, n} h_{i} .
$$

THEOREM. Let the coefficients in the differential equation have bounded third derivatives and assume that (1.4) holds. Let $u$ be the solution of (1.1)-(1.3) and assume that

$$
u \in L^{\infty}\left(0, T ; H^{6}\right) \text { and } u_{\iota} \in L^{2}\left(0, T ; H^{6}\right) .
$$

Let $U(x, 0)$ be the $\mathfrak{H}_{3}$-interpolant of $f(x)=u(x, 0)$. Then, there exists a unique solution $U$ of the collocation equations (1.6), and $U$ converges to $u$ with an error that can be estimated by (5.15).

The order of convergence of $U$ to $u$ is optimal, given the approximating space $\mathfrak{F}_{3}$; however, the smoothness hypotheses in the above theorem are stronger than would be required from approximation theory alone, since boundedness in $H^{4}$ suffices for interpolation to give $O\left(h^{4}\right)$ in $L^{2}$ and $H^{5}$ is more than enough for $L^{\infty}$. 
Thus, we have not obtained as strong a theorem for the collocation procedure as for the corresponding Galerkin procedure.

Mathematics Department

University of Chicago

Chicago, Illinois 60637

1. Phillip J. Davis, Interpolation and Approximation, Blaisdell, Waltham, Mass., 1963. MR 28 \#393.

2. JiM Douglas, JR. \& TODD DUPONT, "The effect of interpolation of coefficients in nonlinear parabolic Galerkin procedures." (To appear.)

3. J. L. Lions \& E. MAGENES, Problèmes aux limites non homogènes et applications.

Vol. 1, Travaux et Recherches Mathématiques, no. 17, Dunod, Paris, 1968. MR 40 \#12. 1969.

4. B. WENDROFF, First Principles of Numerical Analysis, Addison-Wesley, Reading, Mass.,

5. M. F. Wheeler, Thesis, Rice University, Houston, Texas, 1971; SIAM J. Numer. Anal. (To appear.) 